\title{
CONTINUOUS DERIVATIONS ON BANACH ALGEBRAS
}

\section{A. M. SINCLAIR}

1. Introduction. We show (\$2) that a continuous derivation on a Banach algebra over the real or complex field leaves the primitive ideals of the algebra invariant. The method of proof is similar to that of [5]. In [2] it is shown that every (linear) derivation on a semisimple Banach algebra is continuous. Thus every derivation on a semisimple Banach algebra leaves the primitive ideals of the algebra invariant. It is known that a continuous derivation on a commutative Banach algebra over the complex field has range in the radical of the algebra [7], [3]. By adjoining an identity if necessary and observing that the derivation of the identity is zero, we note that this result may be deduced from Theorem 2.2.

In the study of derivations on a semisimple Banach algebra, it is thus of interest to know how derivations behave on primitive Banach algebras. If $\mathfrak{A}$ is a primitive Banach algebra with a continuous faithful algebraically irreducible representation on a Banach space $\mathfrak{X}$ and $D$ is a continuous derivation on $\mathfrak{A}$, is there a bounded linear operator $B$ on $\mathfrak{X}$ such that $D(x)=x B-B x$ for all $x \in \mathfrak{A}$ ? By [1, Theorem 3, p. 87] and the closed graph theorem, there is such a $B$ if $\mathfrak{A}$ (regarded as an algebra of linear operators on $\mathfrak{X}$ ) contains operators of finite rank. We give (Corollary 3.6) necessary and sufficient conditions for such a $B$ to exist in general. We also discuss (Theorem 3.3) a similar problem in primitive algebras over a field.

I am deeply indebted to my supervisor, Dr. B. E. Johnson, for his advice and encouragement, and to the University of Witwatersrand for a University Council Postgraduate Scholarship.

2. Continuous derivations and primitive ideals. A derivation on an algebra $\mathfrak{A}$ is a linear operator $D$ on $\mathfrak{A}$ satisf ying $D(x y)=x D(y)+D(x) y$ for all $x, y \in \mathfrak{A}$.

2.1 Lemma. Let $D$ be a derivation on an algebra $\mathfrak{A}$. Let $P$ be a two sided ideal in $\mathfrak{A}$ and $x \in P$. Then $D^{n}\left(x^{n}\right)+P=n ! D(x)^{n}+P$ for $n$ $=1,2, \cdots$.

Proof. We first show that $D^{j}\left(x^{k}\right) \in P$ for $0 \leqq j<k$. By repeated application of Leibniz's formula, we obtain $D^{i}\left(x^{k}\right)$ as a finite sum of terms of the form $D^{\epsilon_{1}}(x) \cdot D^{\epsilon_{2}}(x) \cdots \cdots D^{\epsilon_{k}}(x)$ where $\epsilon_{i}$ are nonnega-

Received by the editors July $5,1967$. 
tive integers and $\sum_{i=1}^{k} \epsilon_{i}=j<k$. Thus, in each of these terms, there is at least one factor $x$, so each term is in $P$, and hence $D^{j}\left(x^{k}\right) \in P$.

We prove the lemma by induction on $n$. Suppose that the result is true for $n$. Then, by Leibniz's formula and the above,

$$
\begin{aligned}
D^{n+1}\left(x^{n+1}\right) & =D \cdot D^{n}\left(x^{n} \cdot x\right) \\
& =\sum_{j=0}^{n}\left(\begin{array}{l}
n \\
j
\end{array}\right)\left\{D^{i+1}\left(x^{n}\right) \cdot D^{n-j}(x)+D^{j}\left(x^{n}\right) \cdot D^{n-j+1}(x)\right\} \\
& \in n D^{n}\left(x^{n}\right) \cdot D(x)+D^{n}\left(x^{n}\right) \cdot D(x)+P \\
& \subseteq(n+1) ! D(x)^{n+1}+P .
\end{aligned}
$$

2.2 Theorem. Let $\mathfrak{A}$ be a Banach algebra over the real or complex field and let $D$ be a continuous derivation on $\mathfrak{A}$. Then $D$ leaves the primitive ideals of $\mathfrak{A}$ invariant.

Proof. Let $P$ be a closed two sided ideal in $\mathfrak{A}$. If $x \in P$, then in the Banach algebra $\mathfrak{A} / P$

$$
\begin{gathered}
\left\|(D(x)+P)^{n}\right\| 1 / n=\left\{(n !)^{-1}\left\|D^{n}\left(x^{n}\right)+P\right\|\right\}^{1 / n} \\
\leqq(n !)^{-1 / n}\|D\| \cdot\|x\| \rightarrow 0 \text { as } n \rightarrow \infty
\end{gathered}
$$

Thus $D(x)+P$ is a topologically nilpotent element $[6$, p. 12] in $\mathfrak{A} / P$. From the derivation law satisfied by $D$, it follows that $D(P)+P$ is a two sided ideal in $\mathfrak{A}$. Hence $D(P)+P / P$ is contained in the radical of $\mathfrak{A} / P[6$, Theorem 2.3.5, p. 57]. If $P$ is a primitive ideal, or an intersection of primitive ideals, then $\mathfrak{A} / P$ is semisimple so that $D(P) \subseteq P$.

3. Derivations on primitive algebras. Let $\mathfrak{A}$ be an algebra over a field $\Gamma$ and let $\mathfrak{A}$ have a faithful strictly dense $[1$, p. 26] $[6,2.4 .2]$ (linear) representation as linear operators on a $\Gamma$-vector space $\mathfrak{X}$. Let $D$ be a derivation on $\mathfrak{A}$, and for each $\xi \in \mathfrak{X}$, let

$$
P(\xi)=\{\eta \in \mathfrak{X}: x \eta=0 \quad(x \in \mathfrak{A}, x \xi=D(x) \xi=0)\} .
$$

The following two lemmas will be used in Theorem 3.3.

3.1 Lemma. Let $\xi \in \mathfrak{X}, \eta \in \mathcal{P}(\xi)$ and $x \in \mathfrak{A}$ with $x \xi=0$. Then there is $\alpha \in \Gamma$, depending on $\xi, \eta$ and $x$, such that $x \eta=\alpha D(x) \xi$.

Proof. For each $y \in \mathfrak{A}$ with $y D(x) \xi=0$ we have $y x \xi=0$ and $D(y x) \xi=y D(x) \xi+D(y) x \xi=0$. Thus $y D(x) \xi=0$ implies that $y x \eta=0$, and so $x_{\eta} \in \Gamma D(x) \xi$, proving the lemma.

3.2 Lemma. For each $\xi \in \mathfrak{X}, \odot(\xi)$ has dimension of at most two. 
Proof. Suppose $\nu, \eta \in \mathcal{P}(\xi)$ with $\xi, \nu, \eta$ linearly independent. By the strict density of $\mathfrak{A}$ on $\mathfrak{X}$, we choose $x \in \mathfrak{A}$ satisfying $x \xi=0, x \nu=\nu$, and $x \eta=\eta$. Then there are $\alpha, \beta \in \Gamma$ such that $\nu=x \nu=\alpha D(x) \xi$ and $\eta=x \eta$ $=\beta D(x) \xi$. Since $\nu$ and $\eta$ are nonzero, $\alpha$ and $\beta$ are nonzero. Thus $\alpha^{-1} \nu-\beta^{-1} \eta=0$, contradicting the supposition that $\nu, \eta$ are linearly independent.

I am indebted to Dr. B. E. Johnson for suggestions that considerably shortened the proof of the following result.

3.3 ThEOREM. Let $\mathfrak{A}$ be an algebra over a field $\Gamma$ and let $\mathfrak{A}$ have a faithful strictly dense linear representation on a $\Gamma$-vector space $\mathfrak{X}$. Let $D$ be a nonzero derivation on $\mathfrak{A}$. There is a linear operator $B$ on $\mathfrak{X}$ such that $D(x)=B x-x B$ for all $x \in \mathfrak{A}$ if and only if, for some $\xi \in \mathfrak{X}, \rho(\xi)$ has dimension two.

Proof. If $\mathfrak{X}$ has dimension one, then $\mathfrak{A}=\Gamma I$ and $D(\alpha I)=\alpha D(I)=0$ for all $\alpha \in \Gamma$. The theorem is thus vacuously true when dimension of $\mathfrak{X}$ is one. We assume that the dimension of $\mathfrak{X}$ is greater than one. Suppose that there is a linear operator $B$ on $\mathfrak{X}$ so that $D(x)=B x-x B$ for all $x \in \mathfrak{A}$. If $B=\alpha I$ for some $\alpha \in \Gamma$ and $I$ is the identity operator on $\mathfrak{X}, D=0$. Thus there is $\xi \in \mathfrak{X}$ such that $B \xi \notin \Gamma \xi$. For each $x \in \mathfrak{A}$ with $x \xi=D(x) \xi=0, x B \xi=0$. Therefore $\xi, B \xi \in \odot(\xi)$, which has dimension two by Lemma 3.2.

Conversely we suppose that $\rho(\xi)$ has dimension two. Since $P(0)$ $=\{0\}, \xi$ is not zero. Then there is $\eta \in \mathcal{X}$ so that $\odot(\xi)=\Gamma \xi+\Gamma \eta$ and $\xi, \eta$ are linearly independent. We prove that there is $\alpha \in \Gamma \backslash\{0\}$ such that for each $x \in \mathfrak{A}$ with $x \xi=0, x \eta=\alpha D(x) \xi$.

Let $y \in \mathfrak{A}$ with $y \xi=0$ and $y \eta \neq 0$. By Lemma 3.1, there is $\alpha \in \Gamma$ such that $y \eta=\alpha D(y) \xi$. Since $y \eta \neq 0, \alpha$ is nonzero. Let $z \in \mathfrak{A}$ and $\beta \in \Gamma$ satisfying $z \xi=0, z \eta \neq 0$ and $z \eta=\beta D(z) \xi$. By the strict density of $\mathfrak{A}$ on $\mathfrak{X}$, there is $w \in \mathfrak{A}$ with $w z \eta=y \eta$. Hence $(\alpha y-\beta w z) \xi=0$ and $D(\alpha y-\beta w z) \xi$ $=\alpha D(y) \xi-\beta w D(z) \xi=0$. Since $\eta \in \mathcal{P}(\xi), \alpha y \eta-\beta w z \eta=0$ and therefore $\alpha=\beta$. If $z \in \mathfrak{A}$ and $z \xi=z \eta=0$, then $(y+z) \xi=0,(y+z) \eta \neq 0$ and so $(y+z) \eta=\alpha D(y+z) \xi$. From this and the definition of $\alpha$, we obtain $D(z) \xi=0$.

We normalize the choice of $\eta$ in $\rho(\xi)$ by letting $\nu=-\alpha \eta$. Then for each $x \in \mathfrak{A}$ with $x \xi=0, x \nu=-D(x) \xi$. If $x, z \in \mathfrak{A}$ and $x \xi=z \xi$, then $x \nu+D(x) \xi=z \nu+D(z) \xi$. For each $\mu \in \mathfrak{X}$ we let $B \mu=x \nu+D(x) \xi$ where $x \in \mathfrak{A}$ and $x \xi=\mu$. We note that $B$ is well defined and that $B(\gamma x)=\gamma B x$ for each $\gamma \in \Gamma$ and $x \in \mathfrak{A}$. If $\mu, \zeta \in \mathfrak{X}$, we choose $x, y \in \mathfrak{A}$ so that $x \xi=\mu$ and $y \xi=\zeta$. Then $B(\mu+\zeta)=(x+y) \nu+D(x+y) \xi=B \mu+B \zeta$. Therefore $B$ is a linear operator on $\mathfrak{X}$. Let $\mu \in \mathfrak{X}$ and $x \in \mathfrak{A}$. Choose $y \in \mathfrak{A}$ with $y \xi=\mu$. By definition of $B$ and the choice of $y$, 


$$
\begin{aligned}
(B x-x B) \mu & =x y \nu+D(x y) \xi-x B \mu \\
& =D(x) y \xi+x(y \nu+D(y) \xi-B \mu) \\
& =D(x) \mu .
\end{aligned}
$$

3.4 Remark. There are strictly dense algebras of operators on certain vector spaces with nonzero, nonspatial derivations. Let $\mathfrak{A}$ be the free algebra over the real field (say) generated by the symbols $v$ and $w$. Let $D(v)=w$ and $D(w)=0$, and extend $D$ to $\mathfrak{A}$ by the derivation law. If $\mathfrak{A}$ is represented as in [1, Example 5, p. 36], $D$ is not spatial.

3.5 Remark. We show briefly that if the algebra $\mathfrak{A}$ of 3.3 contains nonzero operators of finite rank, then every derivation on $\mathfrak{A}$ (represented on $\mathfrak{X}$ ) is spatial $[1$, Theorem 3, p. 87]. We assume that $\mathfrak{A}$ contains the identity operator on $\mathfrak{X}$; if not it can be adjoined to $\mathfrak{A}$. By Theorem 3.3, we have only to show that if $\mathcal{P}(\xi)=\Gamma \xi$ for each $\xi \in \mathfrak{X}, D$ $=0$. Using the strict density of $\mathfrak{A}$ on $\mathfrak{X}$, we obtain for each $\xi(\neq 0)$ $\in \mathfrak{X}$ a $y \in \mathfrak{A}$ satisfying $y^{2}=y$ and $y \mathfrak{X}=\Gamma \xi$. Since $\odot(\xi)=\Gamma \xi$, it can be shown that $D(y) \xi \in \Gamma \xi$. From this and the derivation law it follows that $x \xi=0$ implies $D(x) \xi=0$. This may be used to show that if $\eta \in \mathfrak{X}$ and $x \in \mathfrak{A}$ with $x \xi=\eta$, then $D(x) \xi \in \Gamma \eta$, and that if $\eta \in \Gamma \xi$, then $D(x) \xi=0$. Replacing $x$ by $x-\gamma I$ for each $\gamma \in \Gamma$, we obtain $D(x) \xi$ $=D(x-\gamma I) \xi \in \Gamma(\eta-\gamma \xi)$. From this it follows that $D(x) \xi=0$ when $\xi, \eta$ are linearly independent. This shows that $D(x) \xi=0$ for each $x \in \mathfrak{A}$ and $\xi \in \mathfrak{X}$.

An elegant proof of this may be given by adapting the proof of [4, Theorem 9, p. 855].

3.6 CoRollary. Let $\mathfrak{A}$ be a primitive Banach algebra over $\boldsymbol{C}$ with a faithfully continuous algebraically irreducible representation on a Banach space $\mathfrak{X}$. Let $D$ be a nonzero derivation on $\mathfrak{A}$. There is a bounded linear operator $B$ on $\mathfrak{X}$ such that $D(x)=B x-x B$ for all $x \in \mathfrak{A}$ if and only if, for some $\xi \in \mathfrak{X}, \odot(\xi)$ has dimension two.

Proof. By Theorem 3.3, and [6, Corollary 2.4.7, p. 62], we have only to show that if $D(x)=B x-x B$ for all $x \in \mathfrak{A}$, where $B$ is a linear operator on $\mathfrak{X}$, then $B$ is continuous. By [2, Remark 4.3], $D$ is continuous on $\mathfrak{A}$. Fix $\eta(\neq 0) \in \mathfrak{X}$ and for each $\xi \in \mathfrak{X}$ let $|\xi|$ $=\inf \{\|x\|: x \in \mathfrak{A}, x \eta=\xi\}$, where $\|\cdot\|$ is the norm on $\mathfrak{A}$. By [6, Theorem 2.2.7, p. 53], $|\cdot|$ is equivalent to the given norm on $\mathfrak{X}$, so we have only to prove the continuity of $B$ in $|\cdot|$. Let $z \in \mathfrak{A}$ with $z \eta=B \eta$. By subtracting the inner derivation on $\mathfrak{A}$ given by $z$, we may assume that $B \eta=0$. For each $\nu \in \mathfrak{X}$ and $x \in \mathfrak{A}$ with $x \eta=\nu, B \nu=B x \eta-x B \eta=D(x) \eta$. 
Thus

$$
\begin{aligned}
|B \nu| & =\inf \{\|y\|: y \in \mathfrak{A}, y \eta=B \nu\} \\
& \leqq \inf \{\|D(x)\|: x \in \mathfrak{A}, x \eta=\nu\} \\
& \leqq\|D\| \cdot|\nu| .
\end{aligned}
$$

\section{REFERENCES}

1. N. Jacobson, Structure of rings, Amer. Math. Soc. Colloq. Publ., Vol. 37, Amer. Math. Soc., Providence, R. I., 1956.

2. B. E. Johnson and A. M. Sinclair, Continuity of derivations and a problem of Kaplansky, Amer. J. Math. (to appear).

3. I. Kaplansky, "Derivations of Banach algebras," in Seminars on analytic functions, Vol. 2, Princeton Univ. Press, Princeton, N. J., 1958.

4. - Modules over operator algebras, Amer. J. Math. 75 (1953), 839-858.

5. D. C. Kleinecke, On operator commutators, Proc. Amer. Math. Soc. 8 (1957), 535-536. 1960.

6. C. E. Rickart, General theory of Banach algebras, Van Nostrand, New York,

7. I. M. Singer and J. Wermer, Derivations on commutative normed algebras, Math. Ann. 129 (1955), 260-264.

The University, Newcastle Upon Tyne, England 